\title{
INFORMATION: PRICE AND IMPACT ON GENERAL WELFARE AND OPTIMAL INVESTMENT. AN ANTICIPATIVE STOCHASTIC DIFFERENTIAL GAME MODEL
}

\author{
CHRISTIAN-OLIVER EWALD, * University of Sydney \\ YAJUN XIAO, ${ }^{* *}$ University of Freiburg
}

\begin{abstract}
Within an anticipative stochastic calculus framework, we study a market game with asymmetric information and feedback effects. We derive necessary and sufficient criteria for the existence of Nash equilibria and study how general welfare is affected by the level of information. In particular, we show that, under certain conditions in a competitive environment, an increased level of information may in fact lower the level of general welfare, leading to the so-called Hirshleifer effect (see Hirshleifer (1971)). Finally, we determine equilibrium prices for particular pieces of information, by extending our market game with a pre-stage, in which information is traded.
\end{abstract}

Keywords: Information; financial market; stochastic differential game

2010 Mathematics Subject Classification: Primary 91B28

Secondary $60 \mathrm{H} 07 ; 60 \mathrm{H} 10 ; 60 \mathrm{H} 30$

\section{Introduction}

In the classical Black-Scholes model and in fact in most other continuous-time financial market models it is assumed that agents' behavior does not influence asset prices and all agents possess the same level of information. These models have been very successfully applied to classical questions such as the pricing of options and derivatives as well as optimal asset allocation. However, these models are not suitable to explain how the level of information influences the general performance of the stock market or how and for what price information may be exchanged between individual agents in the market. There is no doubt that in real-world markets agents possess different levels of information and that it is important to understand what value particular pieces of information have and how general welfare is affected by these. In the first part of this paper, we set up a continuous-time market model in which agents are assumed to influence asset prices and are exposed to different information flows. The framework is that of a stochastic differential game with anticipative strategy sets. We derive necessary and sufficient conditions for the existence of Nash equilibria for this game and characterize these for various levels of information asymmetry. Furthermore, we study the consequences an increased level of information has on general welfare. Information asymmetry is not a contradiction to the efficient market hypotheses, as the agents may indeed learn all the necessary information by carefully studying the market, if they invest enough effort to do so. However, the emphasis here

Received 4 May 2010; revision received 19 November 2010.

* Postal address: School of Mathematics and Statistics, University of Sydney, Sydney, NSW 2006, Australia.

Email address: christian.ewald@sydney.edu.au

** Postal address: Institute for Research in Economic Evolution, University of Freiburg, Platz der Alten Synagoge, KGII 79098 Freiburg i. Br., Germany. 
is in the effort. Different agents invest different amounts of effort or capital in order to obtain information, which may enable them to trade more successfully. Information is costly, and in the second part of this paper we study the aspect of pricing information within a competitive market. To illustrate this, let us consider the following three scenarios. In scenario 1 a private investor may start buying stocks or funds without knowing anything about the market and not intending to learn anything about it, simply because he/she has read an advertisement in their local bank. In scenario 2 a private investor is strongly engaged in monitoring his/her individual portfolio, buying financial newspapers, investing time to watch business channels, etc. Finally, in scenario 3, a big company who represents a significant market player may invest large sums in hiring a consulting company, which essentially provides it with key information on the market. All three scenarios present different aspects under which information may be traded between market players. In the first scenario the exchange of information is costless for the investor while the bank bears costs due to the advertisement campaign. Obviously, there is a matter of trust here, but we leave this issue aside. In scenario 2 the investor invests money and time to obtain information on the market, essentially to trade more successfully and outperform other market participants. The exchange of information in this case is costly for the private investor, while the seller of information, i.e. financial press, media, but also financial institutions, which often provides media with important information on their business strategies, gain from this sale. The situation in the third scenario is very similar to that in the second scenario, as far as the investor is concerned. Here, again, the investor invests to obtain information from the consulting company, with the exchange of information being costly. There is a significant difference in this scenario however, and this reflects the point of view of the seller of information. While in the second scenario the private investor is assumed to be a price taker and has no influence on market prices, a major company which owns large portfolios represents a trader, which has market impact and may influence market prices due to the mechanisms of supply and demand. The consulting company, which is also engaged in the market, must bear in mind the consequences that giving information to a large trader may change prices and, therefore, affect the value of their own portfolios, when considering whether or not and for which price to sell information. The first scenario more or less represents a problem in finding the right advertisement strategy for the bank and can be addressed in the general context of advertisement. A situation as described in scenario 2 has been addressed in continuous-time diffusion-type market models, complete and incomplete, by various authors, for example, Karatzas and Pikovsky (1996), Corcuera et al. (2004), Imkeller (2003), León et al. (2003), and Ewald (2005). In this context the value of additional information is determined from the point of view of a representative uninformed agent. This agent would buy the information for any price $P$ such that

$$
u\left(x, \hat{\pi}^{*}\right) \leq u\left(x-P, \pi^{*}\right),
$$

where $\pi^{*}$ and $\hat{\pi}^{*}$ denote the optimal portfolios under additional and, respectively, no additional information. Here $u$ denotes expected utility from terminal wealth while $x$ and $x-P$ respectively denote the different levels of initial wealth. The owner of the information would sell for any price, as he/she does not fear for any consequences on the market. Any positive price offer for the information would give an incentive to sell, and in the presence of many possible information providers, the seller's price reduces to 0 . In the third scenario, which is the focus of this paper, the situation is more complex, as the seller of the information must take the buyers market impact into account. Selling information comes with the risk that the buyer may use the information in a way that changes market prices to the disadvantage of the seller. This situation has not been studied before in a continuous-time diffusion-type financial market model. The 
framework we consider is most general. We study a market model in which two agents use information flows modeled by filtrations $g^{1}$ and $g^{2}$ in order to buy or sell assets, whose prices they may influence depending on their current position in the market. These filtrations are assumed to satisfy the usual conditions; see Karatzas and Schreve (1988, p. 10). The technical framework of the second part of this paper is an extension of the market game studied in the first part, including two initial stages in which information can be traded for monetary units. We solve for the Nash equilibria of this game and in this way determine competitive prices for the pieces of information sold. In both cases, extended and original market games, agents face continuous-time investment decisions. Trading strategies need to be integrated with respect to price processes in order to compute returns. In diffusion-type models integration with respect to price processes is essentially the same as integration with respect to Brownian motion. The standard stochastic integral, which is the Itô integral, does not allow the integrand to depend on more information than revealed by the Brownian motion itself. In our framework, where agents have asymmetric information, which may exceed the level of information revealed by the underlying Brownian motion, the Itô integral is too restrictive. In order to avoid these problems, we use an anticipative stochastic calculus which has been developed in the last two decades. We use the technical framework based on the forward integral as found in Kohatsu-Higa and Sulem (2006). In order to provide analytically tractable examples, we also make use of the classical technique of enlargement of filtration, developed originally by Jacod (1985), which is nowadays used throughout the literature.

The paper is organized as follows. We give a short introduction of anticipative stochastic calculus in Section 2, while in Section 3 we set up our market model and compute Nash equilibrium strategies. In this section we also study the questions of how these equilibria change with respect to changes in the information level and how general welfare is affected by this. In Section 4 we extend the game with a prestage in which information may be exchanged in return for monetary units and determine equilibrium prices for the information. Section 5 contains the main conclusions from the paper.

\section{A brief review of anticipative stochastic calculus}

In this section we introduce some preliminaries about anticipative stochastic calculus, which is in fact strongly related to what is called Malliavin calculus. A standard reference for this is Nualart (1995). Let us consider the set $\&$ of cylindrical functionals $F: \Omega \rightarrow \mathbb{R}$, given by $F=f\left(\mathbb{W}\left(t_{1}\right), \ldots, \mathbb{W}\left(t_{l}\right)\right)$, where $f \in C_{b}^{\infty}\left(\left(\mathbb{R}^{n}\right)^{l}\right)$ is a smooth function with bounded derivatives of all orders and $(\mathbb{W}(t))$ denotes an $n$-dimensional Brownian motion on $\Omega$. We define the Malliavin derivative operator on $\&$ via

$$
D_{s} F:=\sum_{i=1}^{l} \frac{\partial f}{\partial x_{i}}\left(\mathbb{W}_{t_{1}}(\omega), \ldots, \mathbb{W}_{t_{l}}(\omega)\right) \mathbf{1}_{\left[0, t_{i}\right]}(s),
$$

where $\partial f / \partial x_{i}$ denotes the gradient of $f$ with respect to its $i$ th $n$-dimensional argument. This operator and the iterated operators $D^{k}$ are closable and unbounded from $L^{p}(\Omega)$ into $L^{p}(\Omega \times$ $[0, T]^{k}, \mathbb{R}^{n}$ ) for all $k \geq 1$. Their respective domains are denoted by $\mathbb{D}^{k, p}$ and obtained as the closure of $\&$ with respect to the norms defined by

$$
\|F\|_{k, p}^{p}=\|F\|_{L^{p}(\Omega)}^{p}+\sum_{j=1}^{k}\left\|D^{j} F\right\|_{L^{p}\left(\Omega \times[0, T]^{j}, \mathbb{R}^{n}\right)}^{p} .
$$


The adjoint of the Malliavin derivative operator $D: \mathbb{D}^{1,2} \rightarrow L^{2}\left(\Omega \times[0, T], \mathbb{R}^{n}\right)$ is called the Skorokhod integral and denoted with $\delta$. This operator has the property that its domain contains the class $L_{a}^{2}\left(\Omega \times[0, T], \mathbb{R}^{n}\right)$ of square integrable adapted stochastic processes and its restriction to this class coincides with the Itô integral. We will make use of the notation $\delta(u)=\int_{0}^{T} u_{t} \mathrm{~d} \mathbb{W}_{t}$. The Malliavin derivative operator and the Skorokhod integral are related by the following integration by parts formula:

$$
\mathrm{E}(\delta(u) F)=\mathrm{E}\left(\int_{0}^{T} D_{t} F u(t) \mathrm{d} t\right) \quad \text { for any } F \in \mathbb{D}^{1,2} .
$$

The following proposition is used to calculate the logarithmic derivative, often called information drift in information theory. It will prove particularly useful in our examples in the next section. The result is well known in the case where the underlying process $X$ is a Brownian motion. Even though this is precisely the case which we refer to in our application, we include a more general result here, where $X$ is assumed to be a general time-homogeneous diffusion. This proves to be useful in the framework of stochastic volatility models, where additional information is determined by the level of volatility in the future; see, for example, Ewald (2005).

Proposition 1. Suppose that $X=X\left(T_{0}\right), T_{0} \geq T$, where $X$ solves the stochastic differential equation

$$
\mathrm{d} X(t)=b(X(t)) \mathrm{d} t+\sigma(X(t)) \mathrm{d} \mathbb{W}(t),
$$

with $\mathbb{W}(t)$ a one-dimensional Brownian motion. We assume that the transition density $p(t, u$, $x, y)$ is two times continuously differentiable with respect to $x$ and one time continuously differentiable with respect to t. (Conditions for this in terms of the Malliavin derivative are given in Theorem 2.1.4 and Corollary 2.1.2 of Nualart (1995).) Then

$$
\tilde{\mathbb{W}}(t)=\mathbb{W}(t)-\int_{0}^{t} \sigma(X(u)) \partial_{x} \log \left(p\left(u, T_{0}, X(u), X\left(T_{0}\right)\right)\right) \mathrm{d} u
$$

is a Brownian motion with respect to $g=\left(g_{t}\right)$ with $g_{t}=\mathcal{F}_{t} \vee \sigma\left(X\left(T_{0}\right)\right)$ for $t \leq T$.

Proof. Let $f$ be a smooth function, and let $M$ be an $\mathcal{F}_{s}$-adapted random variable. Then

$$
\begin{aligned}
\mathrm{E}\left((\mathbb{W}(t)-\mathbb{W}(s)) M f\left(X\left(T_{0}\right)\right)\right) & =\mathrm{E}\left(\mathrm{E}\left((\mathbb{W}(t)-\mathbb{W}(s)) M f\left(X\left(T_{0}\right)\right) \mid \mathcal{F}_{t}\right)\right) \\
& =\mathrm{E}\left((\mathbb{W}(t)-\mathbb{W}(s)) M \int f(y) p\left(t, T_{0}, X(t), y\right) \mathrm{d} y\right) \\
& =\mathrm{E}\left(\delta\left(\mathbf{1}_{(s, t]}(u)\right) M \int f(y) p\left(t, T_{0}, X(t), y\right) \mathrm{d} y\right) \\
& =\mathrm{E}\left(\int_{s}^{t} D_{u}\left(M \int f(y) p\left(t, T_{0}, X(t), y \mathrm{~d} y\right) \mathrm{d} u\right) .\right.
\end{aligned}
$$

Because $M$ is adapted to $\mathscr{F}_{s}$, we have $D_{u} M=0$ for $u>s$. Applying first the product rule to $\left.M \int f(y) p\left(u, T_{0}, X(u), y\right) \mathrm{d} y\right)$ and then Fubini's theorem, we obtain

$$
\begin{aligned}
\mathrm{E}\left((\mathbb{W}(t)-\mathbb{W}(s)) M f\left(X\left(T_{0}\right)\right)\right) & =\mathrm{E}\left(\int_{s}^{t} M \int f(y) D_{u} p\left(t, T_{0}, X(t), y\right) \mathrm{d} y \mathrm{~d} u\right) \\
& =\mathrm{E}\left(\int f(y) M \int_{s}^{t} D_{u} p\left(t, T_{0}, X(t), y\right) \mathrm{d} u \mathrm{~d} y\right) .
\end{aligned}
$$


It follows from the Itô formula that

$$
\begin{aligned}
& D_{u} p\left(t, T_{0}, X(t), y\right) \\
&=D_{u}\left\{p(s, X(s), y)+\int_{s}^{t}\left[\frac{\partial p\left(v, T_{0}, X(v), y\right)}{\partial v}+b(X(v)) \frac{\partial p\left(v, T_{0}, X(v), y\right)}{\partial x}\right.\right. \\
&\left.\quad+\frac{1}{2} \sigma^{2}(X(v)) \frac{\partial^{2} p\left(v, T_{0}, X(v), y\right)}{\partial x^{2}}\right] \mathrm{d} v \\
&\left.+\int_{s}^{t} \sigma(X(v)) \frac{\partial p\left(v, T_{0}, X(v), y\right)}{\partial x} \mathrm{~d} \mathbb{W}(v)\right\} .
\end{aligned}
$$

From the Kolmogorov backward equation we can conclude that the expression in the square brackets is 0 . Furthermore, $D_{u} p(s, X(s), y)=0$ for the reason that $u \geq s$ implies that $p(s, X(s), y)$ is $\mathcal{F}_{u}$-adapted. We therefore find that $\mathrm{E}\left((\mathbb{W}(t)-\mathbb{W}(s)) M f\left(X\left(T_{0}\right)\right)\right)$ is given by the expression

$$
\mathrm{E}\left(\int f(y) M \int_{s}^{t} D_{u}\left[\int_{s}^{t} \sigma(X(v)) \frac{\partial p\left(v, T_{0}, X(v), y\right)}{\partial x} \mathrm{~d} \mathbb{W}(v)\right] \mathrm{d} u \mathrm{~d} y\right) .
$$

Applying the Malliavin derivative operator to the expression in the square brackets leads to, according to Nualart (1995, Lemma 1.3.4),

$$
\begin{aligned}
& \mathrm{E}\left((\mathbb{W}(t)-\mathbb{W}(s)) M f\left(X\left(T_{0}\right)\right)\right) \\
&=\mathrm{E}\left(\int f(y) M \int_{s}^{t}(\right. \sigma(X(u)) \frac{\partial p\left(u, T_{0}, X(u), y\right)}{\partial x} \\
&\left.\left.\quad+\int_{u}^{t} D_{u}\left[\sigma(X(v)) \frac{\partial p\left(v, T_{0}, X(v), y\right)}{\partial x}\right] \mathrm{d} \mathbb{W}(v)\right) \mathrm{d} u \mathrm{~d} y\right) .
\end{aligned}
$$

Using the Fubini theorem to interchange the order of integration and taking expectations inside the integral, and, furthermore, realizing that the expectation of an Itô integral with respect to Brownian motion is always 0 , we obtain

$$
\mathrm{E}\left((\mathbb{W}(t)-\mathbb{W}(s)) M f\left(X\left(T_{0}\right)\right)\right)=\mathrm{E}\left(\int f(y) M \int_{s}^{t} \sigma(X(u)) \frac{\partial p\left(u, T_{0}, X(u), y\right)}{\partial x} \mathrm{~d} u \mathrm{~d} y\right) .
$$

Another application of Fubini's theorem and the fact that, for a positive differentiable function $\alpha(x)$, we have $(\partial \log (\alpha(x)) / \partial x) \alpha(x)=\partial \alpha(x) / \partial x$ yields

$$
\begin{aligned}
& \mathrm{E}\left((\mathbb{W}(t)-\mathbb{W}(s)) M f\left(X\left(T_{0}\right)\right)\right) \\
& \quad=\mathrm{E}\left(\int_{s}^{t}\left(\int f(y) M \sigma(X(u)) \frac{\partial \log p\left(u, T_{0}, X(u), y\right)}{\partial x} p\left(u, T_{0}, X(u), y\right) \mathrm{d} y\right) \mathrm{d} u\right) .
\end{aligned}
$$

By the definition of the transition density function we conclude that

$$
\begin{aligned}
& \mathrm{E}\left((\mathbb{W}(t)-\mathbb{W}(s)) M f\left(X\left(T_{0}\right)\right)\right) \\
& \quad=\mathrm{E}\left(f\left(X\left(T_{0}\right)\right) M \int_{s}^{t} \sigma(X(u)) \frac{\partial \log p\left(u, T_{0}, X(u), X\left(T_{0}\right)\right)}{\partial x} \mathrm{~d} u\right) .
\end{aligned}
$$


A density argument then establishes that

$$
\mathrm{E}\left(\mathbb{W}(t)-\mathbb{W}(s)-\int_{s}^{t} \sigma(X(u)) \frac{\partial \log p\left(u, T_{0}, X(u), X\left(T_{0}\right)\right)}{\partial x} \mathrm{~d} u \mid g_{s}\right)=0 .
$$

Now, by the definition of $\tilde{\mathbb{W}}(t)$, the last equality is equivalent to

$$
\mathrm{E}\left(\tilde{\mathbb{W}}(t)-\tilde{\mathbb{W}}(s) \mid g_{s}\right)=0
$$

and $(\tilde{\mathbb{W}}(t))_{[0, T]}$ is therefore a continuous martingale with respect to the filtration $g_{\tilde{\sim}}$ Its quadratic variation is given by $\langle\tilde{\mathbb{W}}(t)\rangle=t$ for $t \in[0, T]$. Hence, by Lévy's theorem, $(\tilde{\mathbb{W}}(t))_{[0, T]}$ is a Brownian motion with respect to $g_{t}$.

Example 1. Assume that $X_{i}\left(T_{0}\right)=\mathbb{W}_{i}\left(T_{0}\right), i=1, \ldots, n$, such that

$$
\mathcal{g}_{t}=\mathcal{F}_{t} \vee \sigma\left(X_{1}\left(T_{0}\right)\right) \vee \cdots \vee \sigma\left(X_{n}\left(T_{0}\right)\right)
$$

We can then explicitly write down the transition probability density of $X_{i}\left(T_{0}\right)$ conditional on $\mathcal{F}_{t}$ :

$$
p\left(t, T_{0}, X_{i}(t), y\right)=\frac{1}{\sqrt{2 \pi\left(T_{0}-t\right)}} \exp \left(-\frac{\left(y-X_{i}(t)\right)^{2}}{2\left(T_{0}-t\right)}\right) .
$$

Then

$$
\sigma\left(X_{i}(u)\right) \partial_{x} \log \left(p\left(u, T_{0}, X_{i}(u), X\left(T_{0}\right)\right)=\frac{X_{i}\left(T_{0}\right)-X_{i}(u)}{T_{0}-u}=\frac{\mathbb{W}_{i}\left(T_{0}\right)-\mathbb{W}_{i}(u)}{T_{0}-u},\right.
$$

and

$$
\tilde{\mathbb{W}}_{i}(t)=\mathbb{W}_{i}(t)-\int_{0}^{t} \frac{\mathbb{W}_{i}\left(T_{0}\right)-\mathbb{W}_{i}(u)}{T_{0}-u} \mathrm{~d} u
$$

is a $g$-Brownian motion, noting that

$$
\mathrm{E}\left(X_{i}(t)-X_{i}(s) \mid g_{s}\right)=\mathrm{E}\left(X_{i}(t)-X_{i}(s) \mid \mathcal{F}_{s} \vee \sigma\left(X_{i}(s)\right)\right) .
$$

In the following section we will use the so-called forward integral, which allows us more flexibility in the choice of stochastic integrands. For details, see, for example, Russo and Valois (1993).

Definition 1. Let $\varphi: z[0, T] \times \Omega \rightarrow \mathbb{R}^{n}$ be a measurable process. The forward integral of $\varphi$ with respect to $\mathbb{W}(t)$ is defined by

$$
\int_{0}^{T} \varphi(t) \cdot \mathrm{d}^{-} \mathbb{W}(t)=\lim _{\varepsilon \rightarrow 0} \int_{0}^{T} \varphi^{\top}(t) \cdot \frac{\mathbb{W}(t+\varepsilon)-\mathbb{W}(t)}{\varepsilon} \mathrm{d} t,
$$

if the limit exists in probability.

The forward integral is related to the Skorokhod integral in the following way. Suppose that $\varphi$ is $\mathbb{R}^{n}$-valued as in Definition 1 with $\varphi \in \mathbb{D}^{1,2}$ satisfying

$$
\mathrm{E}\left(\int_{0}^{T}|\varphi(t)|^{2}\right) \mathrm{d} t+\mathrm{E}\left(\int_{0}^{T} \int_{0}^{T}\left\|D_{s} \varphi(t)\right\|^{2}\right) \mathrm{d} s \mathrm{~d} t \leq \infty
$$


where $\|\cdot\|$ denotes the Euclidean matrix norm. Moreover, assume that

$$
\operatorname{tr}\left(D_{t^{+}} \varphi(t)\right):=\lim _{s \rightarrow t^{+}} \operatorname{tr}\left(D_{s} \varphi(t)\right)
$$

exists in $L^{2}([0, T] \times \Omega)$. Then $\varphi$ is forward integrable and

$$
\int_{0}^{T} \varphi(t) \cdot \mathrm{d}^{-} \mathbb{W}(t)=\delta(\varphi(t))+\int_{0}^{T} \operatorname{tr}\left(D_{t^{+}} \varphi(t) \mathrm{d} t .\right.
$$

A proof of this result can be found in, for example, Russo and Valois (1993) or Kohatsu-Higa and Sulem (2006). Taking into account the fact that the expectation of a Skorokhod integral always vanishes, we obtain

$$
\mathrm{E}\left(\int_{0}^{T} \varphi(t) \cdot \mathrm{d}^{-} \mathbb{W}(t)\right)=\mathrm{E}\left(\int_{0}^{T} \operatorname{tr}\left(D_{t^{+}} \varphi(t) \mathrm{d} t\right) .\right.
$$

Furthermore, it can be shown that if $\varphi$ is forward integrable and càglàd (i.e. left continuous with left limits) and $\Delta:=\left\{0=t_{0}<t_{1}<\cdots<t_{n}=T\right\}$ is a sequence of partitions such that $\Delta_{n}:=\sup _{i=0, \cdots, n-1}\left\{t_{i+1}-t_{i}\right\}$ goes to 0 when $n \rightarrow \infty$, then

$$
\int_{0}^{T} \varphi(t) \cdot \mathrm{d}^{-} \mathbb{W}(t)=\lim _{\Delta_{n} \rightarrow 0} \sum_{i=0}^{n-1} \varphi^{\top}\left(t_{i}\right) \cdot\left(\mathbb{W}\left(t_{i+1}\right)-\mathbb{W}\left(t_{i}\right)\right)
$$

if the limit exists in probability. Taking the latter into account we can indeed argue that the forward integral is predestined to model financial markets in continuous time when allowing trading strategies to depend on a more general information structure. It also follows from the latter equation that in the case where $\mathbb{W}$ remains a semimartingale when changing the filtration, the forward integral coincides with the Itô integral for semimartingales.

\section{Continuous-time market games with heterogeneous information}

We consider a market with a finite time horizon $[0, T]$ and agents which are heterogeneously informed. For simplicity, we restrict the number of agents to two. Our analysis however can easily be modified to model the case of arbitrarily many agents. Assets include one riskless asset, which we call the bond and denote with $B(t)$, and $n$ risky assets, which we think of as stocks and denote with $S^{i}(t)$. The different levels of information are modeled using four different filtrations throughout the remainder of this paper. These are $g^{1}=\left(g_{t}^{1}\right)$ for agent number one, $g^{2}=\left(g_{t}^{2}\right)$ for agent number two, $\mathcal{F}=\left(\mathscr{F}_{t}\right)$, the $\sigma$-algebra generated by the underlying noise process, which we assume to be a Brownian motion $\mathbb{W}(t)$, and, finally, the filtration $g=\left(g_{t}\right)$ for the coefficients of the underlying model. We assume that $\mathcal{F}_{t} \subseteq g_{t}^{p} \subseteq \mathcal{F}_{T}$ for $p=1,2$ and $\mathcal{F}_{t} \subseteq g_{t} \subseteq g_{t}^{1} \cap g_{t}^{2}$ for $t \in[0, T]$. The latter relationship guarantees that even though agents may have different levels of information, they both understand how the market works and how other agents' behavior affects the market. At the current moment we do not impose any further relationships between $g^{1}$ and $g^{2}$. The agents' investments are described by their individual portfolio processes $\pi_{p}=\left(\pi_{p}^{i}(t)\right)_{1 \leq i \leq n}$, where $\pi_{p}^{i}(t)$ denotes the proportion of wealth of agent $p$ which at time $t$ is invested in stock $i=1, \ldots, k$, while the remaining portion $\pi_{p}^{0}(t)$ is assumed to be invested in the bond. We will later model the process of selling pieces of information from one agent to another. In order that the selling agent is not indifferent to 
giving away information to another agent for free, we need to assume that the agents' behavior affects asset prices. More precisely, we assume the following dynamics for assets:

$$
\begin{aligned}
\mathrm{d} B(t) & =r(t) B(t) \mathrm{d} t, \quad B(t)=1, \\
\mathrm{~d} S(t) & =\operatorname{diag}(S(t))\left\{\mu\left(t, \pi_{1}(t), \pi_{2}(t)\right) \mathrm{d} t+\sigma(t) \mathrm{d}^{-} \mathbb{W}(t)\right\}, \quad S(0)>0,
\end{aligned}
$$

where $\operatorname{diag}(S(t))$ is the $n \times n$ matrix with diagonal elements $S^{i}(t)$ and 0 s elsewhere. We assume that the following conditions hold for the coefficients.

(C1) $\mu(t, x, y)=\left(\mu_{i}(t, x, y)_{1 \leq i \leq n}\right)$ is a $g$-adapted process with values in $C\left(\mathbb{R}^{n} \times \mathbb{R}^{n}, \mathbb{R}^{n}\right)$, $r(t)$ is a $g$-adapted and real-valued stochastic process, $\sigma(t)=\left(\sigma_{i j}(t)\right)_{1 \leq i, j \leq n}$ is a $g$-adapted and $\mathbb{R}^{n \times n}$-valued stochastic process.

(C2) $\int_{0}^{T}\left(|r(t)|+|\mu(t, x, y)|+\left\|\sigma(t) \sigma^{\top}(t)\right\|\right) \mathrm{d} t<\infty$ almost surely (a.s.) for all $x, y \in \mathbb{R}^{n}$.

(C3) $\sigma(t)$ is forward integrable and càglàd.

The chosen dynamics (7) incorporate a supply and demand feature, in which agents' current positions influence the growth rates of the asset prices. A similar dynamic for the case of a representative agent has been used in Kohatsu-Higa and Sulem (2006). We denote with $X_{p}\left(t, \pi_{1}, \pi_{2}\right)$ the discounted wealth process corresponding to agent $p$ given chosen investment strategies $\pi_{1}$ and $\pi_{2}$. The wealth processes also depend on the initial endowments of the agents, but, for the moment, we omit this from the notation. The stochastic differential equation governing the evolution of the wealth processes is given by

$$
\mathrm{d} X_{p}\left(t, \pi_{1}, \pi_{2}\right)=X_{p}\left(t, \pi_{1}, \pi_{2}\right)\left(\pi_{p}^{\top}(t)\left(\mu\left(t, \pi_{1}(t), \pi_{2}(t)\right)-r(t)\right) \mathrm{d} t+\pi_{p}^{\top}(t) \sigma(t) \mathrm{d}^{-} \mathbb{W}(t)\right),
$$

with initial condition $X_{p}(0)=x_{p}$. Note that this equation presents a stochastic differential equation with anticipating coefficients. Nevertheless, the Itô formula for the forward integral (see Russo and Vallois (2000)) implies that (8) is satisfied by

$$
\begin{aligned}
X_{p}\left(t, \pi_{1}, \pi_{2}\right)=x_{p} \exp ( & \int_{0}^{T}\left(\pi_{p}^{\top}(s)\left(\mu\left(s, \pi_{1}(s), \pi_{2}(s)\right)-r(s)\right)\right. \\
& \left.\left.\quad-\frac{1}{2} \pi_{p}^{\top}(s) \sigma(s) \sigma^{\top}(s) \pi_{p}(s)\right) \mathrm{d} s+\int_{0}^{T} \pi_{p}^{\top}(s) \sigma(s) \mathrm{d}^{-} \mathbb{W}(s)\right) .
\end{aligned}
$$

For technical reasons, we have to impose certain restrictions on our portfolio strategies which guarantee that the solution above is well defined.

Definition 2. We call a pair of portfolio strategies $\left(\pi_{1}, \pi_{2}\right)$ admissible and write $\left(\pi_{1}, \pi_{2}\right) \in \mathcal{A}$ if the following conditions are satisfied.

(i) $X_{p}\left(t, \pi_{1}, \pi_{2}\right)>0$ for all $t \in[0, T]$.

(ii) $\pi_{p}(t)$ is càglàd and $\pi_{p}^{\top}(t) \sigma(t)$ is forward integrable. Moreover,

$$
\int_{0}^{T}\left\{\left|\pi_{p}^{\top}(t) \mu\left(t, \pi_{1}(t), \pi_{2}(t)\right)-r(t)\right|+\left|\pi_{p}^{\top}(t) \sigma(t) \sigma^{\top}(t) \pi_{p}(t)\right|\right\} \mathrm{d} t<\infty .
$$

(iii) For any bounded càglàd process $\tilde{\pi}$ such that $\tilde{\pi}^{\top}(t) \sigma(t)$ is forward integrable, there exists a $\gamma>0$ such that the families

$$
\left\{\left|M_{1}\left(T, \pi_{1}+\varepsilon \tilde{\pi}, \pi_{2}\right)\right|\right\}_{0 \leq \varepsilon \leq \gamma} \quad \text { and } \quad\left\{\left|M_{2}\left(T, \pi_{1}, \pi_{2}+\varepsilon \tilde{\pi}\right)\right|\right\}_{0 \leq \varepsilon \leq \gamma}
$$


are uniformly integrable, where

$$
\begin{aligned}
M_{p}\left(t, \pi_{1}, \pi_{2}\right):=\mathrm{E}\left(\int_{0}^{t}(\right. & \mu\left(s, \pi_{1}(s), \pi_{2}(s)\right)-r(s)+J_{\mu}^{\pi_{p}}(s) \pi_{p}(s) \\
& \left.\left.\quad-\sigma(s) \sigma^{\top}(s) \pi_{p}(s)\right) \mathrm{d} s+\int_{0}^{t} \sigma(s) \mathrm{d}^{-} \mathbb{W}(s) \mid g_{t}^{p}\right) .
\end{aligned}
$$

Here $J_{\mu}^{\pi_{p}}(s)$ is the Jacobian matrix of $\mu$ with respect to $\pi_{p}$ evaluated at time $s$.

We assume that our agents are risk averse and that their objective is to maximize the expected utility from discounted terminal wealth. In order to obtain analytically tractable results, we use logarithmic utility. Taking this into account, the payoffs for the agents in our market game are given by

$$
u_{p}\left(\pi_{1}, \pi_{2}\right):=\mathrm{E}\left(\ln \left(X_{p}^{\pi_{1}, \pi_{2}}(T)\right)\right)
$$

for $p=1,2$. We note that the payoffs $u_{p}$ also depend on the initial endowments $x_{1}$ and $x_{2}$, but we omit this in our notation. In this setup the optimization objective for both agents is identical and, therefore, asymmetry effects concerning the level of risk averseness are left out in our discussion. The asymmetry arising in our model comes from the fact that the strategies of the individual players rely on different information and that they may effect the market in different ways. We consider the market to be in equilibrium if the strategy pair $\left(\pi_{1}^{*}, \pi_{2}^{*}\right) \in \mathcal{A}$ constitutes a Nash equilibrium, i.e.

$$
\begin{aligned}
& u_{1}\left(\pi_{1}^{*}, \pi_{2}^{*}\right)=\sup _{\pi_{1} \in \mathcal{A}_{1}\left(\pi_{2}^{*}\right)} \mathrm{E}\left(\ln \left(X_{1}^{\pi_{1}, \pi_{2}^{*}}(T)\right)\right), \\
& u_{2}\left(\pi_{1}^{*}, \pi_{2}^{*}\right)=\sup _{\pi_{2} \in \mathcal{A}_{2}\left(\pi_{1}^{*}\right)} \mathrm{E}\left(\ln \left(X_{1}^{\pi_{1}^{*}, \pi_{2}}(T)\right)\right),
\end{aligned}
$$

with $A_{1}\left(\pi_{2}^{*}\right)=\left\{\pi_{1} \mid\left(\pi_{1}, \pi_{2}^{*}\right) \in \mathcal{A}\right\}$ and $A_{2}\left(\pi_{1}^{*}\right)=\left\{\pi_{2} \mid\left(\pi_{1}^{*}, \pi_{2}\right) \in \mathcal{A}\right\}$. The following theorem provides necessary and sufficient conditions on the existence of a Nash equilibrium for the market game above in terms of a martingale condition.

Theorem 1. Under the assumptions stated in the preceding paragraph, the following statements hold.

(i) If $\left(\pi_{1}^{*}, \pi_{2}^{*}\right)$ constitutes a Nash equilibrium for the market game then $M_{p}\left(t, \pi_{1}^{*}, \pi_{2}^{*}\right)$ for $t \in[0, T]$ is a martingale with respect to the filtration $\mathrm{g}^{p}$ for $p=1,2$.

(ii) If $\left(\pi_{1}^{*}, \pi_{2}^{*}\right) \in \mathcal{A}$ and $M\left(t, \pi_{1}^{*}, \pi_{2}^{*}\right), t \in[0, T]$, is a martingale with respect to the filtration $\mathrm{g}^{p}$ and $u_{p}\left(\pi_{1}, \pi_{2}\right)$ is concave with respect to $\pi_{p}$ for $p=1,2$, then $\left(\pi_{1}^{*}, \pi_{2}^{*}\right)$ constitutes a Nash equilibrium for the market game.

Proof. (i) If $\left(\pi_{1}^{*}, \pi_{2}^{*}\right)$ constitutes a Nash equilibrium for the market game then, for bounded $\theta_{1}$ as in Definition 2(iii), we have

$$
u_{1}\left(\pi_{1}^{*}, \pi_{2}^{*}\right) \geq u_{1}\left(\pi_{1}^{*}+\varepsilon \theta_{1}, \pi_{2}^{*}\right)
$$

for all $\varepsilon$ in an open neighborhood of 0 . This implies that the partial directional derivative of $u_{1}$ 
along the direction $\theta_{1}$ evaluated at $\pi_{1}^{*}$ is 0 , i.e.

$$
\begin{aligned}
& 0=\left.\frac{\mathrm{d}}{\mathrm{d} \varepsilon} u_{1}\left(\pi_{1}^{*}+\varepsilon \theta_{1}, \pi_{2}^{*}\right)\right|_{\varepsilon=0} \\
&=\mathrm{E}\left(\int_{0}^{T} \theta_{1}^{\top}(t)\left(\mu\left(t, \pi_{1}^{*}(t), \pi_{2}^{*}(t)\right)-r(t)+J_{\mu}^{\pi_{1}^{*}}(t) \pi_{1}(t)-\sigma(t) \sigma^{\top}(t) \pi_{1}^{*}(t)\right) \mathrm{d} t\right. \\
&\left.\quad+\int_{0}^{T} \theta_{1}^{\top}(t) \sigma(s) \mathrm{d}^{-} \mathbb{W}(s)\right) .
\end{aligned}
$$

Note that the differentiation and the integral can be interchanged because our admissibility definition implies that $\left\{\left|M_{1}\left(T, \pi_{1}^{*}+\varepsilon \theta_{1}, \pi_{2}^{*}\right)\right|\right\}_{0 \leq \varepsilon \leq \gamma}$ is uniformly integrable. Let us now consider the particular process $\theta_{1}(u)=\theta(t) \mathbf{1}_{(t, t+h]}(u), h>0,0 \leq t \leq T$, where $\theta(t)$ is a bounded, $\mathbb{R}^{n}$-valued, and $g_{t}^{1}$-measurable random variable. Thus, (13) can be written as

$$
\begin{aligned}
0=\mathrm{E}\left(\theta ^ { \top } ( t ) \left(\int_{t}^{t+h}(\right.\right. & \mu\left(u, \pi_{1}^{*}(u), \pi_{2}^{*}(u)\right)-r(u)+J_{\mu}^{\pi_{1}^{*}}(u) \pi_{1}(u) \\
& \left.\left.\left.-\sigma(u) \sigma^{\top}(u) \pi_{1}(u)\right) \mathrm{d} u+\int_{t}^{t+h} \sigma(u) \mathrm{d}^{-} \mathbb{W}(u)\right)\right) .
\end{aligned}
$$

Since (14) holds for all such $\theta$, we conclude that

$$
\begin{aligned}
& 0=\mathrm{E}\left(\int_{t}^{t+h}\left(\mu\left(u, \pi_{1}^{*}(u), \pi_{2}^{*}(u)\right)-r(u)+J_{\mu}^{\pi_{1}^{*}}(u) \pi_{1}(u)-\sigma(u) \sigma^{\top}(u) \pi_{1}(u)\right) \mathrm{d} u\right. \\
& \left.\quad+\int_{t}^{t+h} \sigma(u) \mathrm{d}^{-} \mathbb{W}(u) \mid g_{t}^{1}\right) .
\end{aligned}
$$

Using the definition of $M_{p}\left(t, \pi_{1}, \pi_{2}\right)$, we obtain

$$
\mathrm{E}\left(M_{1}\left(t+h, \pi_{1}^{*}, \pi_{2}^{*}\right)-M_{1}\left(t, \pi_{1}^{*}, \pi_{2}^{*}\right) \mid g_{t}^{1}\right)=0 .
$$

An analogous argumentation using $u_{2}\left(\pi_{1}^{*}, \pi_{2}^{*}\right) \geq u_{2}\left(\pi_{1}^{*}, \pi_{2}^{*}+\varepsilon \theta_{2}\right)$ establishes

$$
\mathrm{E}\left(M_{2}\left(t+h, \pi_{1}^{*}, \pi_{2}^{*}\right)-M_{2}\left(t, \pi_{1}^{*}, \pi_{2}^{*}\right) \mid g_{t}^{1}\right)=0 .
$$

From (16) and (17), we infer that $M_{p}\left(t, \pi_{1}^{*}, \pi_{2}^{*}\right)$ is a $g^{p}$-martingale for $p=1,2$.

(ii) Let us now assume that there exists a pair $\left(\pi_{1}^{*}, \pi_{2}^{*}\right)$ such that $M_{1}\left(t, \pi_{1}^{*}, \pi_{2}^{*}\right)$ is a $g^{1}$-martingale and $M_{2}\left(t, \pi_{1}, \pi_{2}\right)$ a $g^{2}$-martingale. Therefore, (16) and (17) hold simultaneously. Let us consider the optimization problem for agent 1. Equation (16) implies that (15) holds; hence, (14) holds for $\theta(t)$ bounded, $\mathbb{R}^{n}$-valued, and $g^{1}$ measurable. Inductively, we see that (13) holds for processes of the form

$$
\tilde{\theta}_{1}(u)=\sum_{i=0}^{n-1} \theta_{1}\left(t_{i}\right) \mathbf{1}_{\left(t_{i}, t_{i+1}\right]}(u), \quad 0=t_{0}<t_{1} \cdots<t_{n}=T,
$$

where the $\theta_{1}\left(t_{i}\right)$ are bounded, $\mathbb{R}^{n}$-valued, and $g_{t_{i}}^{1}$-measurable random variables. Here we use the equality

$$
\int_{0}^{T} \tilde{\theta}_{1}^{\top}(t) \sigma(t) \mathrm{d}^{-} \mathbb{W}(t)=\sum_{i=0}^{n-1} \int_{t_{i}}^{t_{i+1}} \theta_{1}^{\top}\left(t_{i}\right) \sigma(u) \mathrm{d}^{-} \mathbb{W}(u) .
$$


We find that (13) is true for all simple processes $\tilde{\theta}_{1}(u)$ and a density argument establishes that (13) holds for all processes $\theta_{1}$ as in Definition 2(iii). This implies that

$$
\left.\frac{\mathrm{d}}{\mathrm{d} \varepsilon} u_{1}\left(\pi_{1}+\varepsilon \theta_{1}, \pi_{2}\right)\right|_{\varepsilon=0}=0 .
$$

On the other hand, using the fact that $u_{p}\left(\pi_{1}, \pi_{2}\right)$ is concave in each $\pi_{p}$, we obtain

$$
\begin{aligned}
& \frac{1}{\varepsilon}\left(u_{1}\left(\pi_{1}+\varepsilon \theta_{1}, \pi_{2}\right)-u_{1}\left(\pi_{1}, \pi_{2}\right)\right) \\
& \quad=\frac{1}{\varepsilon}\left(u_{1}\left((1-\varepsilon) \frac{\pi_{1}}{1-\varepsilon} \varepsilon \theta_{1}, \pi_{2}\right)-u_{1}\left(\pi_{1}, \pi_{2}\right)\right) \\
& \quad \geq \frac{1}{\varepsilon}\left((1-\varepsilon) u_{1}\left(\frac{\pi_{1}}{1-\varepsilon}, \pi_{2}\right)+\varepsilon u_{1}\left(\theta_{1}, \pi_{2}\right)-u_{1}\left(\pi_{1}, \pi_{2}\right)\right) \\
& \quad=\frac{1}{\varepsilon}\left(u_{1}\left(\frac{\pi_{1}}{1-\varepsilon}, \pi_{2}\right)-u_{1}\left(\pi_{1}, \pi_{2}\right)\right)+u_{1}\left(\theta_{1}, \pi_{2}\right)-u_{1}\left(\frac{\pi_{1}}{1-\varepsilon}, \pi_{2}\right) .
\end{aligned}
$$

Taking the limit as $\varepsilon \rightarrow 0$, and taking into account the fact that

$$
\lim _{\varepsilon \rightarrow 0} \frac{1}{\varepsilon}\left(u_{1}\left(\frac{\pi_{1}^{*}}{1-\varepsilon}, \pi_{2}^{*}\right)-u_{1}\left(\pi_{1}^{*}, \pi_{2}^{*}\right)\right)=0,
$$

as the latter is basically the directional derivative of $u_{1}$ along $\pi_{1}^{*}$, which by (18) must be 0 , we obtain $0 \geq u_{1}\left(\theta_{1}, \pi_{2}^{*}\right)-u_{1}\left(\pi_{1}^{*}, \pi_{2}^{*}\right)$. As $\theta_{1}$ can be chosen within the set $\mathcal{A}_{1}\left(\pi_{2}^{*}\right)$, we obtain, by formally setting $\theta_{1}=\pi_{1}$,

$$
u_{1}\left(\pi_{1}^{*}, \pi_{2}^{*}\right) \geq u_{1}\left(\pi_{1}, \pi_{2}^{*}\right) \text { for all } \pi_{1} \in \mathcal{A}_{1}\left(\pi_{2}^{*}\right) .
$$

Analogously, we obtain

$$
u_{2}\left(\pi_{1}^{*}, \pi_{2}^{*}\right) \geq u_{2}\left(\pi_{1}^{*}, \pi_{2}\right) \text { for all } \pi_{2} \in \mathcal{A}_{2}\left(\pi_{1}^{*}\right) .
$$

This means that $\left(\pi_{1}^{*}, \pi_{2}^{*}\right)$ is a Nash equilibrium for the market game.

In the following we discuss how we can use the criterion presented in Theorem 1 in order to identify Nash equilibria for our market game with heterogeneous information.

Lemma 1. Assume that $\left(\pi_{1}^{*}, \pi_{2}^{*}\right)$ is a Nash equilibrium for our market game. Then the process $\varepsilon \mapsto \mathrm{E}\left(\int_{t}^{t+\varepsilon} \sigma(u) \mathrm{d}^{-} \mathbb{W}(u) \mid g_{t}^{p}\right)$ has absolutely continuous paths for $p=1,2$ and the derivative

$$
I_{p}(t):=\frac{\mathrm{d}}{\mathrm{d} \varepsilon} \mathrm{E}\left(\int_{t}^{t+\varepsilon} \sigma(u) \mathrm{d}^{-} \mathbb{W}(u) \mid g_{t}^{p}\right)
$$

exist a.s. for $p=1,2$. Furthermore, the following equation holds for $p=1,2$ :

$$
\mathrm{E}\left(\mu\left(t, \pi_{1}^{*}(t), \pi_{2}^{*}(t)\right)-r(t)+J_{\mu}^{\pi_{p}^{*}}(t) \pi_{p}(t)-\sigma(t) \sigma^{\top}(t) \pi_{p}(t) \mid g_{t}^{p}\right)+I_{p}(t)=0 .
$$

Under the concavity assumption for the utilities in Theorem 1(ii), condition (22) is a sufficient condition for a pair $\left(\pi_{1}^{*}, \pi_{2}^{*}\right) \in \mathcal{A}$ to constitute a Nash equilibrium.

Proof. These statements follow from (15) when dividing the latter by $h$ and taking the limit as $h \rightarrow 0$, Theorem 1, and Definition 2 . 
For the choice of $\mu$, various specifications appear to be reasonable. However, to obtain tractable dynamics and analytical results, we focus on the linear form

$$
\mu\left(t, \pi_{1}, \pi_{2}\right)=\mu(t)+a(t) \pi_{1}+b(t) \pi_{2} .
$$

For the general case, the latter should be considered as a first-order approximation. In order to satisfy condition (C1), we need to assume that the processes $\mu(t), a(t)$, and $b(t)$ are $g$-adapted. In order to apply the second part of Theorem 1 , it is important to note that, under our assumptions, a sufficient criterion for concavity of $u_{p}\left(\pi_{1}, \pi_{2}\right)$ is the following.

Assumption 1. Suppose that $\left(a(t)+a^{\top}(t)\right)-\sigma(t) \sigma^{\top}(t)$ and $\left(b(t)+b^{\top}(t)\right)-\sigma \sigma^{\top}(t)$ take values in the set of negative definite matrices.

The latter assumption can be interpreted in a way that the influences of both agents' portfolio strategies on expected returns are embedded in the noise. Otherwise, the agents may drive the stock prices arbitrarily high just by buying and selling large volumes to obtain a high return. Obviously, embedded does not mean without effect. We will assume from now on that Assumption 1 is satisfied. For notational reasons, let us define the matrix-valued function

$$
\Sigma: M^{n \times n} \rightarrow M^{n \times n}, \quad y \mapsto \sigma \sigma^{\top}-2 y .
$$

Using this specification, we obtain the following characterization of a Nash equilibrium.

Proposition 2. If the system of equations

$$
\begin{aligned}
& \pi_{1}^{*}(t)=\Sigma^{-1}(a(t))\left[\mu(t)-r(t)+I_{1}(t)+b(t) \mathrm{E}\left(\pi_{2}^{*}(t) \mid g_{t}^{1}\right)\right], \\
& \pi_{2}^{*}(t)=\Sigma^{-1}(b(t))\left[\mu(t)-r(t)+I_{2}(t)+a(t) \mathrm{E}\left(\pi_{1}^{*}(t) \mid g_{t}^{2}\right)\right]
\end{aligned}
$$

admits a solution $\left(\pi_{1}^{*}, \pi_{2}^{*}\right) \in \mathcal{A}$, then $\left(\pi_{1}^{*}, \pi_{2}^{*}\right)$ constitutes a Nash equilibrium for our market game.

Proof. This follows directly from Lemma 1, noting that $J_{\mu}^{\pi_{1}}=a(t), J_{\mu}^{\pi_{2}}=b(t)$, and that all coefficients are measurable with respect to $g_{t}^{p}, p=1,2$.

To better understand how the equilibrium strategies are constructed, we study how they change, while changing the complexity of the model, starting with the standard Black-Scholes model, in which we have standard information and no market impact, i.e. $g_{t}^{p}=\mathscr{F}_{t}$ for $t \in[0, T]$ and $a(t)=b(t)=0$. In this case $I_{1}(t)=I_{2}(t)=0$, as the expectation of an Itô integral is always 0 and, therefore, the equilibrium strategies are just the Merton rule. If we allow for market impact but no nonstandard information, we will still have $I_{1}(t)=I_{2}(t)=0$; however, the equilibrium strategies now adjust for the actions of the opponent. In this case agents have complete information about the opponent's strategies and the Nash equilibrium is given by

$$
\begin{aligned}
& \pi_{1}^{*}(t)=\Sigma^{-1}(a(t))\left[\mu(t)-r(t)+b(t) \pi_{2}^{*}(t)\right], \\
& \pi_{2}^{*}(t)=\Sigma^{-1}(a(t))\left[\mu(t)-r(t)+a(t) \pi_{1}^{*}(t)\right] .
\end{aligned}
$$

Now, in the presence of nonstandard, possibly asymmetric information two things occur. First, the agents are no longer able to fully reflect on their opponent's strategy and instead have to take expectations based on their current level of information. This amounts to taking conditional expectations in Proposition 2. Furthermore, additional $I_{p}(t)$ terms occur. These 
can be interpreted as information drifts and adjust the strategy for a differently perceived growth rate of the underlying asset.

The particular form of the equilibrium strategies in Proposition 2 is implicit and has been chosen in order to understand how agents react and adjust to their opponent's strategies. Substituting the formula for $\pi_{2}^{*}(t)$ into the formula for $\pi_{1}^{*}(t)$, and vice versa, it is possible to obtain an explicit form. Note that in order to obtain explicit formulae for the strategies, it is only necessary to give explicit formulae for the conditional expectations on the right-hand sides of the expressions in Proposition 2. Such expressions are derived in the following proposition.

Proposition 3. Assume that $\left(\pi_{1}^{*}, \pi_{2}^{*}\right)$ constitutes a Nash equilibriumfor our market game. Then the conditional expectations $\mathrm{E}\left(\pi_{1}^{*}(t) \mid g_{t}^{2}\right)$ and $\mathrm{E}\left(\pi_{2}^{*}(t) \mid g_{t}^{1}\right)$ in Proposition 2 are explicitly given by the formulae

$$
\begin{aligned}
& \mathrm{E}(\left.\pi_{1}^{*}(t) \mid g_{t}^{2}\right) \\
&=\left(\mathbf{1}-\Sigma^{-1}(a(t)) b(t) \Sigma^{-1}(b(t)) a(t)\right)^{-1} \\
& \quad \times\left\{\Sigma^{-1}(a(t))\left(\mu(t)-r(t)+\tilde{I}_{1}(t)\right)+\Sigma^{-1}(a(t)) b(t) \Sigma^{-1}(b(t))\left(\mu(t)-r(t)+\tilde{I}_{2}(t)\right)\right\}, \\
& \mathrm{E}\left(\pi_{2}^{*}(t) \mid g_{t}^{1}\right) \\
&=\left(\mathbf{1}-\Sigma^{-1}(b(t)) a(t) \Sigma^{-1}(a(t)) b(t)\right)^{-1} \\
& \quad \times\left\{\Sigma^{-1}(b(t))\left(\mu(t)-r(t)+\tilde{I}_{2}(t)\right)+\Sigma^{-1}(b(t)) a(t) \Sigma^{-1}(a(t))\left(\mu(t)-r(t)+\tilde{I}_{1}(t)\right)\right\},
\end{aligned}
$$

where $\tilde{I}_{1}(t)=\mathrm{E}\left(I_{1}(t) \mid g_{t}^{2}\right)$ and $\tilde{I}_{2}(t)=\mathrm{E}\left(I_{2}(t) \mid g_{t}^{1}\right)$ denote the information drift of the individual agents as perceived by the opposite agent.

Proof. The proof follows from Proposition 2 by substituting the equation for $\pi_{2}^{*}(t)$ into the equation for $\pi_{1}^{*}(t)$, and vice versa, and then taking conditional expectations on $g_{t}^{1}$ and, respectively, $g_{t}^{2}$.

For the case of nonstandard homogeneous information, we obtain the following corollary.

Corollary 1. Assume that both agents have the same level of information, i.e. $g^{1}=g^{2}$. If the system of equations

$$
\begin{aligned}
& \pi_{1}^{*}(t)=\Sigma^{-1}(a(t))\left(\mu(t)-r(t)+I(t)+b(t) \pi_{2}^{*}(t)\right), \\
& \pi_{2}^{*}(t)=\Sigma^{-1}(b(t))\left(\mu(t)-r(t)+I(t)+a(t) \pi_{1}^{*}(t)\right),
\end{aligned}
$$

where $I(t)=I_{1}(t)=I_{2}(t)$, admits a solution $\left(\pi_{1}^{*}, \pi_{2}^{*}\right) \in \mathcal{A}$, then $\left(\pi_{1}^{*}, \pi_{2}^{*}\right)$ constitutes a Nash equilibrium for the corresponding market game.

Proof. Symmetry of information implies that the conditional expectation in Proposition 2 can be replaced by the actual strategies. Furthermore, from the definition, it is clear that $I_{1}(t)=I_{2}(t)$.

In the case above explicit solutions can be obtained simply by substituting the expressions for $\pi_{2}^{*}$ and $\pi_{1}^{*}$ into $\pi_{1}^{*}$ and $\pi_{2}^{*}$, respectively, and solving out. Let us now assume that the agents do not only have the same level of information, but also that the market impact of both agents is the same. This relates to choosing $a(t)=b(t)$. In this case we are particularly interested in symmetric Nash equilibria.

Corollary 2. Under symmetric information and the same market impact factors $a(t)=b(t)$, a symmetric Nash equilibrium $\left(\pi^{*}, \pi^{*}\right)$ of our market game is given by

$$
\pi^{*}(t)=\left(\mathbf{1}-\Sigma^{-1}(a(t)) a(t)\right)^{-1} \cdot \Sigma^{-1}(a(t))(\mu(t)-r(t)+I(t)) .
$$


Proof. The proof follows by straightforward computation from Corollary 1 .

In the following we study the welfare implications of information in our market game. By welfare implications we mainly mean whether the market is better of with more information or not. Doing an analysis starting with a representative agent model, such as in the classical literature Karatzas and Pikovsky (1996), Corcuera et al. (2004), Imkeller (2003), León et al. (2003), and Ewald (2005), the answer to this question is trivial: yes! In our framework the analysis however is different, in the way that given more information the agents may be able to outperform and in fact harm each other, with more severe consequences. We will derive explicit conditions on the model parameters which determine whether general welfare is improved or worsened by adding more information. In order to proceed with this, we need the following technical lemma.

Lemma 2. Assume that a Nash equilibrium for our market game exists. Then $\int_{0}^{t} \sigma(s) \mathrm{d}^{-} \mathbb{W}(s)$ is a $g^{p}$-semimartingale for $p=1,2$. Furthermore, if, additionally, the matrix-valued process $\sigma(s)$ is invertible a.s. then $\mathbb{W}$ is a $g^{p}$-semimartingale.

Proof. Under our assumption that the process $\sigma(s)$ is $g$-adapted, we find that the forward integral $\int_{0}^{t} \sigma(s) \mathrm{d}^{-} \mathbb{W}(s)$ is $g_{t}^{p}$-adapted. From (10) we obtain

$$
\begin{aligned}
\int_{0}^{t} \sigma(s) \mathrm{d}^{-} \mathbb{W}(s)= & M_{p}\left(t, \pi_{1}, \pi_{2}\right) \\
& -\mathrm{E}\left(\int _ { 0 } ^ { t } \left(\mu(s)+a(s) \pi_{1}(s)+b(s) \pi_{2}(s)-r(s)+a(s) \pi_{p}(s)\right.\right. \\
& \left.\left.-\sigma(s) \sigma^{\top}(s) \pi_{p}(s)\right) \mathrm{d} s \mid g_{t}^{p}\right) .
\end{aligned}
$$

By separating the positive and negative parts of the integrands in the conditional expectation, the latter can clearly be written as the difference of two nondecreasing $g^{p}$-adapted processes. On the other hand, it follows from Proposition 1 and (10) that $M_{p}\left(t, \pi_{1}, \pi_{2}\right)$ is a continuous martingale. According to Definition 3.1 of Karatzas-Schreve (1988), $\int_{0}^{t} \sigma(s) \mathrm{d}^{-} \mathbb{W}(s)$ is therefore a continuous semimartingale.

For the following discussion, we assume that $g=\mathcal{F}$. Let us consider a filtration $\mathscr{H}=\left(\mathscr{H}_{t}\right)$ such that $g_{t} \subset \mathscr{H}_{t}$ for all $t \in[0, T]$. Denote Nash equilibria of our market game corresponding to the setup $g^{1}=g=g^{2}$ by $\left(\hat{\pi}_{1}^{*}, \hat{\pi}_{2}^{*}\right)$ and Nash equilibria corresponding to the setup $g^{1}=$ $\mathscr{H}=g^{2}$ by $\left(\pi_{1}^{*}, \pi_{2}^{*}\right)$.

Definition 3. The information $\mathscr{H}=\left(\mathscr{H}_{t}\right)$ is welfare increasing, if the payoffs from $\left(\pi_{1}^{*}, \pi_{2}^{*}\right)$ Pareto dominate the payoffs from $\left(\hat{\pi}_{1}^{*}, \hat{\pi}_{2}^{*}\right)$. Here $\mathscr{H}$ is called welfare decreasing if the opposite is true. Furthermore, we define the information welfare impact of $\mathscr{H}$ as the vector

$$
\operatorname{iwi}(\mathscr{H})=\left(\begin{array}{l}
u_{1}\left(\pi_{1}^{*}, \pi_{2}^{*}\right)-u_{1}\left(\hat{\pi}_{1}^{*}, \hat{\pi}_{2}^{*}\right) \\
u_{2}\left(\pi_{1}^{*}, \pi_{2}^{*}\right)-u_{2}\left(\hat{\pi}_{1}^{*}, \hat{\pi}_{2}^{*}\right)
\end{array}\right) .
$$

Clearly, the information welfare impact does not depend on the initial wealth, and information is welfare increasing if both components are positive and welfare decreasing if both components are negative. We have the following proposition, which provides necessary and sufficient conditions depending on the various parameters of the game, whether or not information is welfare increasing. 
Proposition 4. Writing $\mathbb{W}(t)=\widehat{\mathbb{W}}(t)-\int_{0}^{t} \alpha(s) \mathrm{d} s$ according to Lemma 2 with $\hat{\mathbb{W}}(t)$ an $\mathcal{H}$-Brownian motion and using the notation

$$
\begin{aligned}
& \Sigma_{1}=\Sigma(a(t))-b(t) \Sigma(b(t))^{-1} \Sigma(a(t)), \\
& \Sigma_{2}=\Sigma((t))-b(t) \Sigma(b(t))^{-1} \Sigma(a(t)),
\end{aligned}
$$

the two components of the information welfare impact of $\mathscr{H}$ are explicitly given as

$$
\begin{gathered}
\operatorname{iwi}(\mathcal{H})_{1}=\mathrm{E}\left(\int _ { 0 } ^ { T } \left(\left(\Sigma_{1}^{-1} \sigma(t) \alpha(t)\right)^{\top} a \Sigma_{1}^{-1} \sigma(t) \alpha(t)+\left(\Sigma_{1}^{-1} \sigma(t) \alpha(t)\right)^{\top} b \Sigma_{2}^{-1} \sigma(t) \alpha(t)\right.\right. \\
-\frac{1}{2}\left(\Sigma_{1}^{-1} \sigma(t) \alpha(t)\right)^{\top} \sigma(t) \sigma(t)^{\top} \Sigma_{1}^{-1} \sigma(t) \alpha(t) \\
\left.\left.+\left(\Sigma_{1}^{-1} \sigma(t) \alpha(t)\right)^{\top} \sigma^{\top}(t) \alpha(t)\right) \mathrm{d} t\right), \\
\operatorname{iwi}(\mathcal{H})_{2}=\mathrm{E}\left(\int _ { 0 } ^ { T } \left(\left(\Sigma_{2}^{-1} \sigma(t) \alpha(t)\right)^{\top} a \Sigma_{1}^{-1} \sigma(t) \alpha(t)+\left(\Sigma_{2}^{-1} \sigma(t) \alpha(t)\right)^{\top} b \Sigma_{2}^{-1} \sigma(t) \alpha(t)\right.\right. \\
-\frac{1}{2}\left(\Sigma_{2}^{-1} \sigma(t) \alpha(t)\right)^{\top} \sigma(t) \sigma(t)^{\top} \Sigma_{2}^{-1} \sigma(t) \alpha(t) \\
\left.\left.+\left(\Sigma_{2}^{-1} \sigma(t) \alpha(t)\right)^{\top} \sigma^{\top}(t) \alpha(t)\right) \mathrm{d} t\right) .
\end{gathered}
$$

Proof. Using Corollary 1, we can easily derive the following two equations for the equilibrium strategy $\left(\pi_{1}^{*}, \pi_{2}^{*}\right)$ under information $\mathscr{H}$ :

$$
\pi_{1}^{*}=\Sigma_{1}^{-1}(\mu(t)-r(t))+\Sigma_{1}^{-1} I(t), \quad \pi_{2}^{*}=\Sigma_{2}^{-1}(\mu(t)-r(t))+\Sigma_{2}^{-1} I(t) .
$$

The equilibrium strategies without additional information are given by the market impact adjusted Merton rules:

$$
\hat{\pi}_{1}^{*}=\Sigma_{1}^{-1}(\mu(t)-r(t)), \quad \hat{\pi}_{2}^{*}=\Sigma_{2}^{-1}(\mu(t)-r(t)) .
$$

Substitution of these strategies into the utility function leads to the following expression for the first component of iwi $(\mathscr{H})$ :

$$
\begin{aligned}
& \mathrm{E}\left(\int _ { 0 } ^ { T } \left(\Sigma_{1}^{-1}(\mu(t)-r(t))\left(a(t) \Sigma_{1}^{-1} I(t)+b(t) \Sigma_{2}^{-1} I(t)\right)+\left(\Sigma_{1}^{-1} I(t)\right)^{\top}(\mu(t)-r(t))\right.\right. \\
& \quad+\left(\Sigma_{1}^{-1} I(t)\right)^{\top}\left(a \Sigma_{1}^{-1}(\mu(t)-r(t))+a \Sigma_{1}^{-1} I\right) \\
& \quad+\Sigma_{1}^{-1} I\left(b \Sigma_{2}^{-1}(\mu(t)-r(t))+b \Sigma_{2}^{-1} I\right)-\frac{1}{2}\left(\Sigma_{1}^{-1} I\right)^{\top} \sigma(t) \sigma(t)^{\top} \Sigma_{1}^{-1} I \\
& \left.\quad-\frac{1}{2}\left(\Sigma_{1}^{-1} I\right)^{\top} \sigma(t) \sigma(t)^{\top} \Sigma_{1}^{-1} I-\frac{1}{2}\left(\Sigma_{1}^{-1} I\right)^{\top} \sigma(t) \sigma(t)^{\top}\left(\Sigma_{1}^{-1}(\mu(t)-r(t))\right)^{\top}\right) \mathrm{d} t \\
& \left.\quad+\int_{0}^{T}\left(\Sigma_{1}^{-1} I\right) \mathrm{d}^{-} \mathbb{W}(t)\right) .
\end{aligned}
$$

A similar expression can be derived for the second component. Under our assumptions, it follows from Lemma 2 and Biagini and Oksendal (2005, p. 178) that

$$
\mathrm{E}\left(\int_{0}^{t} g(a(s), b(s), \mu(s)-r(s)) I(s) \mathrm{d} s\right)=0
$$

for any bounded function $g$. Using this relationship, it can be verified that the long expression 
above simplifies to

$$
\begin{aligned}
\mathrm{E}\left(\int_{0}^{T}\right. & \left(\left(\Sigma_{1}^{-1} \sigma(t) \alpha(t)\right)^{\top} a \Sigma_{1}^{-1} \sigma(t) \alpha(t)+\left(\Sigma_{1}^{-1} \sigma(t) \alpha(t)\right)^{\top} b \Sigma_{2}^{-1} \sigma(t) \alpha(t)\right. \\
& \left.\left.-\frac{1}{2}\left(\Sigma_{1}^{-1} \sigma(t) \alpha(t)\right)^{\top} \sigma(t) \sigma(t)^{\top} \Sigma_{1}^{-1} \sigma(t) \alpha(t)+\left(\Sigma_{1}^{-1} \sigma(t) \alpha(t)\right)^{\top} \sigma^{\top}(t) \alpha(t)\right) \mathrm{d} t\right) .
\end{aligned}
$$

The analysis of the second component is completely analogous.

The expressions for the information welfare impact are quite lengthy. For the one-dimensional case, we are able to derive the following corollary.

Corollary 3. Under the assumptions that there is only one stock traded at the market, the market parameters are given as $\mu(t) \equiv \mu, a(t) \equiv a$, and $b(t) \equiv b$ with constants $\mu, a, b \in \mathbb{R}$, and that $\Sigma_{\min }:=\min \left\{\Sigma_{1}, \Sigma_{2}\right\}>0$, the information $\mathscr{H}=\left(\mathscr{H}_{t}\right)$ is welfare increasing if

$$
1+\frac{a}{\Sigma_{1}}+\frac{b}{\Sigma_{2}} \geq \frac{\sigma^{2}}{2 \Sigma_{\min }}
$$

and welfare decreasing if

$$
1+\frac{a}{\Sigma_{1}}+\frac{b}{\Sigma_{2}} \leq \frac{\sigma^{2}}{2 \Sigma_{\max }}
$$

with $\Sigma_{\max }:=\max \left\{\Sigma_{1}, \Sigma_{2}\right\}$.

Proof. Under the assumptions in the corollary, it is easy to verify that the components of the information welfare impact vector in Proposition 4 simplify to

$$
\operatorname{iwi}(\mathscr{H})=\left(\begin{array}{c}
\frac{1}{\Sigma_{1}}\left(\frac{a}{\Sigma_{1}}+\frac{b}{\Sigma_{2}}+1-\frac{\sigma^{2}}{2 \Sigma_{1}}\right) \mathrm{E}\left(\int_{0}^{t} \alpha^{2}(t) \mathrm{d} t\right) \\
\frac{1}{\Sigma_{2}}\left(\frac{a}{\Sigma_{1}}+\frac{b}{\Sigma_{2}}+1-\frac{\sigma^{2}}{2 \Sigma_{2}}\right) \mathrm{E}\left(\int_{0}^{t} \alpha^{2}(t) \mathrm{d} t\right)
\end{array}\right) .
$$

The information is welfare increasing if both components of this vector are positive and welfare decreasing if both components are negative. Obviously, we have $\mathrm{E}\left(\int_{0}^{t} \alpha^{2}(t) \mathrm{d} t\right) \geq 0$. The conditions for positivity and, respectively, negativity are therefore exactly as stated in the corollary.

The corollary above specifies a certain region of the parameter space consisting of feasible parameters $\left(a, b, \sigma^{2}\right)$ in which information is welfare increasing. Figure 1 shows this region for the example of initially enlarged filtration.

Note that some of the points in the welfare increasing region may not satisfy the concavity condition, which in this case would correspond to $2 \max \{a, b\} \leq \sigma^{2}$. However, the concavity condition is not necessary for the existence of Nash equilibria, which in our definition of welfare increasing is implicitly assumed. It is worth mentioning though that, in general, the intersection between those points in the welfare increasing region and those points which satisfy the concavity condition is not empty.

In order to demonstrate how our results apply to the classical case of enlarged initial filtration, we include the following example. 


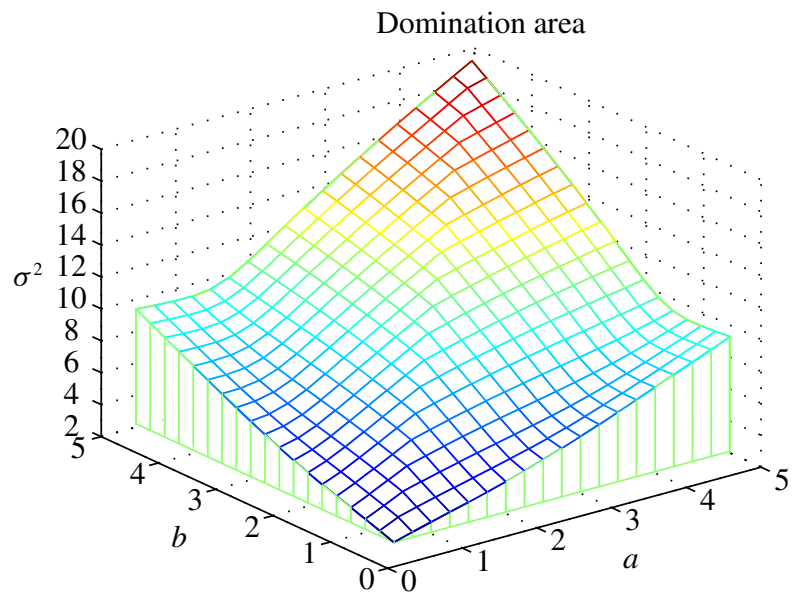

FiguRE 1: Welfare increasing region.

Example 2. Let us study the implications of the various statements above for the case of initially enlarged filtration.

(i) Consider, for $T_{0}>T$, the initially enlarged filtrations $g^{p}=\left(g_{t}^{p}\right)$ with

$$
g_{t}^{p}=\mathcal{F}_{t} \vee \sigma\left(\mathbb{W}_{v_{1}^{p}}\left(T_{0}\right)\right) \vee \cdots \vee \sigma\left(\mathbb{W}_{v_{k p}^{p}}\left(T_{0}\right)\right)
$$

for $t \in[0, T]$, where $\left\{v_{1}^{p}, \ldots, v_{k_{p}}^{p}\right\}$ for $p=1,2$ are subsets of $\{1, \ldots, n\}$. Denote the intersection $\left\{v_{1}^{1}, \ldots, v_{k_{1}}^{1}\right\} \cap\left\{v_{1}^{2}, \ldots, v_{k_{2}}^{2}\right\}$ by $\left\{v_{1}, \ldots, v_{t}\right\}$. This set may possibly be empty. We have

$$
\begin{aligned}
I_{p}(t) & =\left.\frac{\mathrm{d}}{\mathrm{d} \varepsilon} \mathrm{E}\left(\int_{t}^{t+\varepsilon} \sigma(u) \mathrm{d}^{-} \mathbb{W}(u) \mid g_{t}^{p}\right)\right|_{\varepsilon=0} \\
& =\left.\frac{\mathrm{d}}{\mathrm{d} \varepsilon} \mathrm{E}\left(\int_{t}^{t+\varepsilon} \sigma(u) \mathrm{d} \tilde{\mathbb{W}}(u)+\int_{t}^{t+\varepsilon} \sigma(u) \frac{\mathbb{W}\left(T_{0}\right)-\mathbb{W}(u)}{T_{0}-u} \mathrm{~d} u \mid g_{t}^{p}\right)\right|_{\varepsilon=0} \\
& =\sigma(t) \alpha^{p}(t),
\end{aligned}
$$

with

$$
\alpha_{i}^{p}(t)= \begin{cases}\frac{\mathbb{W}_{i}\left(T_{0}\right)-\mathbb{W}_{i}(t)}{T_{0}-t}, & i \in\left\{v_{1}^{1}, \ldots, v_{k_{p}}^{p}\right\} \\ 0, & \text { otherwise. }\end{cases}
$$

This implies that

$$
\tilde{I}_{1}(t)=\mathrm{E}\left(I_{1}(t) \mid g_{2}(t)\right)=\sigma(t) \alpha(t)=\mathrm{E}\left(I_{2}(t) \mid g_{1}(t)\right)=\tilde{I}_{2}(t)
$$

with

$$
\alpha_{i}(t)= \begin{cases}\frac{\mathbb{W}_{i}\left(T_{0}\right)-\mathbb{W}_{i}(t)}{T_{0}-t}, & i \in\left\{v_{1}, \ldots, v_{t}\right\}, \\ 0, & i \notin\left\{s_{1}, \ldots, s_{t}\right\} .\end{cases}
$$


Substitution of these expressions into the corresponding expressions from Proposition 2 and Proposition 3 leads to analytical formulae for the Nash equilibrium. If the additional information is strictly complementary, i.e.

$$
\left\{v_{1}^{1}, \ldots, v_{k_{1}}^{1}\right\} \cap\left\{v_{1}^{2}, \ldots, v_{k_{2}}^{2}\right\}=\varnothing,
$$

we find that $\alpha=0$ and the equilibrium strategies simplify slightly. Such a case is particularly interesting to study from the point of view of cooperative game theory.

(ii) Consider the case where $g^{2}=\mathcal{F}$ and $g^{1}$ is given as the initially enlarged filtration, i.e. $g_{t}^{1}=\mathcal{F}_{t} \vee \sigma\left(\mathbb{W}_{1}\left(T_{0}\right)\right) \vee \cdots \vee \sigma\left(\mathbb{W}_{n}\left(T_{0}\right)\right)$ for all $t \in[0, T]$ and $T_{0}>T$. This distribution of information leads to the Nash equilibria

$$
\begin{aligned}
& \pi_{1}^{*}(t)=\Sigma(a(t))^{-1}\left(\mu(t)-r(t)+b(t) \pi_{2}^{*}+\sigma(t) \frac{\mathbb{W}\left(T_{0}\right)-\mathbb{W}(t)}{T_{0}-t}\right), \\
& \pi_{2}^{*}(t)=\Lambda^{-1}(\mu(t)-r(t))+\Lambda^{-1} a(t) \Sigma(a(t))^{-1}(\mu(t)-r(t)),
\end{aligned}
$$

where $\Lambda=\Sigma(b(t))-a(t) \Sigma(a(t))^{-1} b(t)$. In the case that agent 1 is small and does not have any market impact, i.e. $a(t)=0$, the latter Nash equilibrium simplifies to

$$
\begin{aligned}
& \pi_{1}^{*}(t)=\Sigma^{-1}\left(\mu(t)-r(t)+b(t) \pi_{2}^{*}+\sigma(t) \frac{\mathbb{W}\left(T_{0}\right)-\mathbb{W}(t)}{T_{0}-t}\right), \\
& \pi_{2}^{*}(t)=\Sigma^{-1}(\mu(t)-r(t)) .
\end{aligned}
$$

(iii) Assuming symmetric information and initially enlarged filtrations $\mathcal{g}^{1}=g^{2}=\mathscr{H}$ with

$$
\mathscr{H}_{t}:=\mathcal{F}_{t} \vee \sigma\left(\mathbb{W}_{1}\left(T_{0}\right)\right) \vee \cdots \vee \sigma\left(\mathbb{W}_{n}\left(T_{0}\right)\right), \quad T_{0} \geq T,
$$

as well as symmetric market impact $a(t)=b(t)$, we obtain from the discussion above and Corollary 2 that a symmetric Nash equilibrium is given by

$$
\begin{aligned}
& \pi_{1}^{*}(t)=\Sigma^{-1}(a(t))\left(\mu(t)-r(t)+\sigma(t) \frac{\mathbb{W}\left(T_{0}\right)-\mathbb{W}(t)}{T_{0}-t}+b(t) \pi_{2}^{*}(t)\right), \\
& \pi_{2}^{*}(t)=\Sigma^{-1}(b(t))\left(\mu(t)-r(t)+\sigma(t) \frac{\mathbb{W}\left(T_{0}\right)-\mathbb{W}(t)}{T_{0}-t}+a(t) \pi_{1}^{*}(t)\right) .
\end{aligned}
$$

As indicated before, in this case an explicit solution can be obtained by substitution of the second strategy into the first strategy and vice versa, and solving out.

\section{Trading of information}

In the preceding section we studied how different levels of information affect the equilibrium of the market. While it was assumed that agents have different levels of information, they were not supposed to exchange and share their private information. In this section we will extend our market model in the way that agents are allowed to sell there own private information to their opponent and/or buy the private information of their opponent. For this reason, we extend our original game, which represents a continuous-time sequential game, by two additional stages, which occur before agents invest in the market. For reasons of simplicity, we only treat the case where one of the agents, say agent 1 , is better informed than the other agent, i.e. $g_{t}^{2} \subset g_{t}^{1}$ for 
all $t \in[0, T]$. In the first stage agent 1 announces a price $P$ for which he/she would be willing to sell the information $g^{1}$ to his/her opponent. In the second stage agent 2 decides whether to buy or not to buy the information offered for the price announced by agent 1 . If agent 2 decides to buy information, he/she faces two consequences. First his/her initial wealth is lowered by the amount he/she has to pay for the information. Second, in order to trade on the market and choose a portfolio, agent 2 can now make use of the increased level of information, which is then represented by the information flow $g^{1}$ rather than $g^{2}$. From the perspective of agent 1 , the situation looks as follows. If he/she sells the information, his/her initial wealth will be raised by the amount he/she charges for this information, but he/she faces, as a consequence, his/her opponent now being able to use the increased level of information to decide on his/her individual investment strategy. In a model where agents' behavior does not influence asset prices, the latter would not really lead to consequences and agent 1 would be willing to give away information for free. However, in a model where prices are not determined exogenously, the seller of information has to fear that the buyer of information may use this information in a way that affects asset prices to his/her disadvantage. Summarizing, there are two factors which have to be taken into account in our extended market game when determining the equilibrium and the equilibrium price for the information.

1. Buying information for price $P$ lowers initial wealth from $x$ to $x-P$, but provides the buyer with an increased level of information, which he/she may use to improve his investment strategy and obtain a higher expected return.

2. Selling information for price $P$ increases initial wealth from $x$ to $x+P$, but the agent has to face possible consequences on his/her own optimal investment strategy and expected return due to the increased level of information of his/her opponent.

Both agents have to weigh up the benefits and losses in order to make their decisions.

Definition 4. A price $P^{*}$ is called an equilibrium price for the information $\mathscr{H}=\left(\mathscr{H}_{t}\right)$ if the sequential game described above with the choice of $g^{1}=\mathscr{H}$ has a Nash equilibrium of the type

$\left(\left\{P^{*}, \pi_{1}^{*}\right\},\left\{\right.\right.$ 'buy only if the price is less than or equal to $\left.\left.P^{*}, \pi_{2}^{*}\right\}\right)$.

The definition above guarantees that, if the information is offered at the equilibrium price, it will indeed be traded at that price. In the following we compute equilibrium prices for the case that agent 1 is better informed but does not necessarily have the same market impact. This is the typical setup when a consulting company sells their information and expertise to a client which presents a major market maker. Criteria 1 and 2 above still apply for this setup. In order to solve our extended market game, we apply backward induction. In the previous section we studied the third stage and have identified equilibrium strategies for general levels of information. We found that the equilibrium strategies do not depend on initial wealth. This feature depends on our choice of utility function as the logarithm, but is also observed with other utility functions such as exponential utility. Note, however, that even though the equilibrium strategies are unaffected by the initial wealth, the amount of utility obtained from following these strategies is affected. Using this fact, we find that in the last stage two scenarios are possible. Scenario one occurs if information is traded within the first two stages. In this case both players have the same level of information $g^{1}$ in the last stage. We denote the equilibrium strategies for the corresponding subgame starting in stage 3 with $\left(\pi_{1}^{*}, \pi_{2}^{*}\right)$. If information is not traded within the first two periods then agents possess asymmetric information in the third stage. In this case 
we denote the equilibrium for the corresponding subgame by $\left(\hat{\pi}_{1}^{*}, \hat{\pi}_{2}^{*}\right)$. These equilibria can be computed with the methods presented in the previous section.

Proposition 5. Let $P^{*}$ be a solution of the system

$$
u_{2}^{x_{2}-P^{*}}\left(\pi_{1}^{*}, \pi_{2}^{*}\right)=u_{2}^{x_{2}}\left(\hat{\pi}_{1}^{*}, \hat{\pi}_{2}^{*}\right), \quad u_{1}^{x_{1}+P^{*}}\left(\pi_{1}^{*}, \pi_{2}^{*}\right) \geq u_{1}^{x_{1}}\left(\hat{\pi}_{1}^{*}, \hat{\pi}_{2}^{*}\right) .
$$

Here the upper indexes on the utilities denote the agents' initial endowment. Then $P^{*}$ is an equilibrium price for the information $g^{1}$ in the extended market game illustrated above. In particular, an equilibrium price is unique.

Proof. The proof follows mainly from the discussion above and the definition of an equilibrium price. As the maximal expected utility depends monotonically increasing on the initial wealth and the price $P^{*}$ of the information is added to agent 1 's initial wealth $x_{1}$, agent 1 is trying to set the price as high a possible. Agent 2 has to wager whether to buy or not to buy the information for this price. Obtaining more information raises his/her maximum expected utility, but the price of the information $P^{*}$ is subtracted from his/her initial wealth. The first equality in Proposition 5 sets the price in a way that agent 2 is indifferent about buying or not buying. Even though agent 2 would be willing to buy for a price which satisfies the first equality, it is not a priori clear that agent 1 would sell for this price, as he/she has to wager the consequence of having an opponent on the market which is better informed than original, against the immediate prospect of more initial wealth. This decision is reflected by the inequality in Proposition 5. Using these arguments, it then follows from backward induction that $\left(\left\{P^{*}, \pi_{1}^{*}\right\},\left\{\right.\right.$ 'buy only if the price is less than or equal to $\left.\left.P^{*}, \pi_{2}^{*}\right\}\right)$ is a sequential Nash equilibrium and, therefore, that $P^{*}$ is an equilibrium price.

Definition 5. The price $P^{*}$ is called a feasible price if

$$
u_{2}^{x_{2}-P^{*}}\left(\pi_{1}^{*}, \pi_{2}^{*}\right) \geq u_{2}^{x_{2}}\left(\hat{\pi}_{1}^{*}, \hat{\pi}_{2}^{*}\right), \quad u_{1}^{x_{1}+P^{*}}\left(\pi_{1}^{*}, \pi_{2}^{*}\right) \geq u_{1}^{x_{1}}\left(\hat{\pi}_{1}^{*}, \hat{\pi}_{2}^{*}\right) .
$$

By definition, an equilibrium price is feasible. Feasibility of a price ensures that information is traded under this price; however, the selling agent may perform suboptimally. In the presence of many information providers, feasible prices other than the equilibrium price may occur. It is straightforward to verify that the two inequalities in Definition 5 are equivalent to the following two inequalities:

$$
\begin{gathered}
P^{*} \geq x_{1} \exp \left\{\mathrm { E } \left(\int _ { 0 } ^ { T } \left[\pi_{1}^{*}(t)\left(\mu\left(t, \pi_{1}^{*}, \pi_{2}^{*}\right)-r(t)\right)-\hat{\pi}_{1}^{*}(t)\left(\mu\left(t, \hat{\pi}_{1}^{*}, \hat{\pi}_{2}^{*}\right)-r(t)\right)\right.\right.\right. \\
\left.-\frac{1}{2}\left(\left(\pi_{1}^{*}(t)\right)^{2}-\left(\hat{\pi}_{1}^{*}(t)\right)^{2}\right) \sigma^{2}(t)\right] \mathrm{d} t \\
\left.\left.+\int_{0}^{T}\left(\pi_{1}^{*}(t)-\hat{\pi}_{1}^{*}(t)\right) \sigma(t) \mathrm{d}^{-} \mathbb{W}(t)\right)\right\}-x_{1}, \\
P^{*} \leq x_{2} \exp \left\{\mathrm { E } \left(\int _ { 0 } ^ { T } \left[\pi_{2}^{*}(t)\left(\mu\left(t, \pi_{1}^{*}, \pi_{2}^{*}\right)-r(t)\right)-\hat{\pi}_{2}^{*}(t)\left(\mu\left(t, \hat{\pi}_{1}^{*}, \hat{\pi}_{2}^{*}\right)-r(t)\right)\right.\right.\right. \\
\left.-\frac{1}{2}\left(\left(\pi_{2}^{*}(t)\right)^{2}-\left(\hat{\pi}_{2}^{*}(t)\right)^{2}\right) \sigma^{2}(t)\right] \mathrm{d} t \\
\left.\left.+\int_{0}^{T}\left(\pi_{2}^{*}(t)-\hat{\pi}_{2}^{*}(t)\right) \sigma(t) \mathrm{d}^{-} \mathbb{W}(t)\right)\right\}-x_{2} .
\end{gathered}
$$


Note that we can evaluate the contribution of the forward integral by means of (4):

$$
\mathrm{E}\left(\int_{0}^{T}\left(\pi_{1}^{*}(t)-\hat{\pi}_{1}^{*}\right) \sigma(t) \mathrm{d}^{-} \mathbb{W}(t)\right)=\mathrm{E}\left(\int_{0}^{T} D_{t^{+}}\left(\left(\pi_{1}^{*}(t)-\hat{\pi}_{1}^{*}(t)\right) \sigma(t)\right) \mathrm{d} t\right) .
$$

For an equilibrium price, the second inequality has to be satisfied as an equality. The computation of the price of the information in general can now be done along the following lines. Use the formulae for the equilibrium strategies from the previous sections, substitute these into the formulae above, solve the second formula for $P^{*}$, and see whether the solution verifies the first inequality. If this is the case, $P^{*}$ is the equilibrium price for the information specified. In the following we consider the case of initially enlarged filtration. More precisely, we consider the case of a single stock, and choose $g_{t}^{1}=\mathcal{F}_{t} \vee \sigma\left(W_{T_{0}}\right)$ for $T_{0}>T$ and $g_{t}^{2}=\mathscr{F}_{t}$ for $t \in[0, T]$. It follows from Example 2(ii) and (iii) that

$$
\begin{aligned}
\pi_{1}^{*}(t)= & \frac{\sigma^{2}(t)-b(t)}{\sigma^{4}(t)-2(a(t)+b(t)) \sigma^{2}(t)+3 a(t) b(t)}\left[\mu(t)-r(r)+\sigma(t) \frac{\mathbb{W}_{T_{0}}-\mathbb{W}_{t}}{T_{0}-t}\right], \\
\pi_{2}^{*}(t)= & \frac{\sigma^{2}(t)-a(t)}{\sigma^{4}(t)-2(a(t)+b(t)) \sigma^{2}(t)+3 a(t) b(t)}\left[\mu(t)-r(r)+\sigma(t) \frac{\mathbb{W}_{T_{0}}-\mathbb{W}_{t}}{T_{0}-t}\right], \\
\hat{\pi}_{1}^{*}(t)= & \frac{\sigma^{2}(t)-b(t)}{\sigma^{4}(t)-2(a(t)+b(t)) \sigma^{2}(t)+3 a(t) b(t)}[\mu(t)-r(r)] \\
& +\frac{\sigma(t)}{\sigma^{2}(t)-2 a(t)} \frac{\mathbb{W}_{T_{0}}-\mathbb{W}_{t}}{T_{0}-t}, \\
\hat{\pi}_{2}^{*}(t)= & \frac{\sigma^{2}(t)-a(t)}{\sigma^{4}(t)-2(a(t)+b(t)) \sigma^{2}(t)+3 a(t) b(t)}[\mu(t)-r(r)] .
\end{aligned}
$$

Substituting these strategies into the inequalities above, while using the fact that, for any bounded and measurable function $f$,

$$
\begin{aligned}
& \mathrm{E}\left(\int_{0}^{T} f(\mu(t), r(t), \sigma(t)) \frac{\mathbb{W}_{T_{0}}-\mathbb{W}_{t}}{T_{0}-t} \mathrm{~d} t\right) \\
& \quad=\mathrm{E} \int_{0}^{T} f(\mu(t), r(t), \sigma(t)) \mathrm{d} \tilde{\mathbb{W}}(t)-\mathrm{E}\left(\int_{0}^{T} f(\mu(t), r(t), \sigma(t)) \mathrm{d} \mathbb{W}(t)\right) \\
& \quad=0,
\end{aligned}
$$

we obtain

$$
\begin{aligned}
P^{*} \geq x_{1} \exp \left\{\mathrm { E } \left(\int_{0}^{T}(\right.\right. & \left(a(t)\left(\frac{\sigma^{2}(t)-b(t)}{k}\right)^{2}+b(t) \frac{\left(\sigma^{2}(t)-a(t)\right)\left(\sigma^{2}(t)-b(t)\right)}{k^{2}}\right. \\
& \left.-\frac{a(t)}{\left(\sigma^{2}(t)-2 a(t)\right)^{2}}\right) \\
& -\frac{1}{2}\left(\left(\frac{\sigma^{2}(t)-b(t)}{k}\right)^{2}-\left(\frac{1}{\sigma^{2}(t)-2 a(t)}\right)^{2}\right) \\
+ & \left.\left(\frac{\sigma^{2}(t)-b(t)}{k}-\frac{1}{\sigma^{2}(t)-2 a(t)}\right)\right) \\
& \left.\left.\times \sigma^{2}(t)\left(\frac{\mathbb{W}_{T_{0}}-\mathbb{W}_{t}}{T_{0}-t}\right)^{2} \mathrm{~d} t\right)\right\}-x_{1},
\end{aligned}
$$




$$
\begin{array}{r}
P^{*} \leq x_{2} \exp \left\{\mathrm { E } \left(\int _ { 0 } ^ { T } \left(\left(a(t) \frac{\sigma^{2}(t)-b(t)}{\sigma^{2}(t)-a(t)}+b(t)-\frac{\sigma^{2}(t)}{2}\right) \frac{\left(\sigma^{2}(t)-a(t)\right)^{2}}{k^{2}}\right.\right.\right. \\
\left.\left.\left.+\frac{\sigma^{2}(t)-a(t)}{k}\right) \sigma^{2}(t)\left(\frac{\mathbb{W}_{T_{0}}-\mathbb{W}_{t}}{T_{0}-t}\right)^{2} \mathrm{~d} t\right)\right\}-x_{2} .
\end{array}
$$

Depending on the complexity of the model, the values on the right-hand side of the equalities can either be computed in closed form, using numerical methods, or Monte Carlo valuation. Figures 2- 4 present cases for which we computed feasible price areas under the assumptions above. The equilibrium prices are represented by the upper border of the feasible price areas. The ask price represents the minimum price for which the informed agent would be willing to sell the information, the offer price is the maximum price the uninformed agent would be willing to pay for the information. The feasible price region is indicated with dots. We see that, for the case where the uninformed agent has no market impact, the informed agent would actually be willing to give the information away for free.

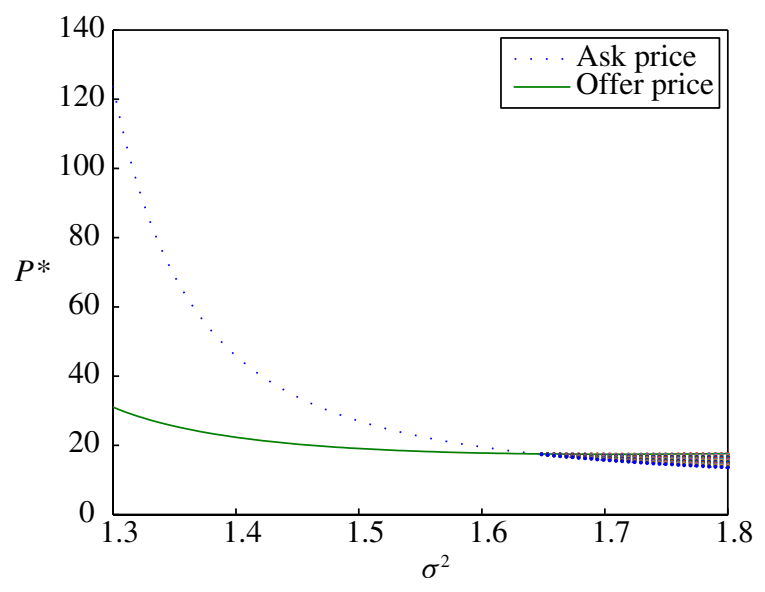

FIGURE 2: Feasible price area for $a=0$ and $b=0.5$.

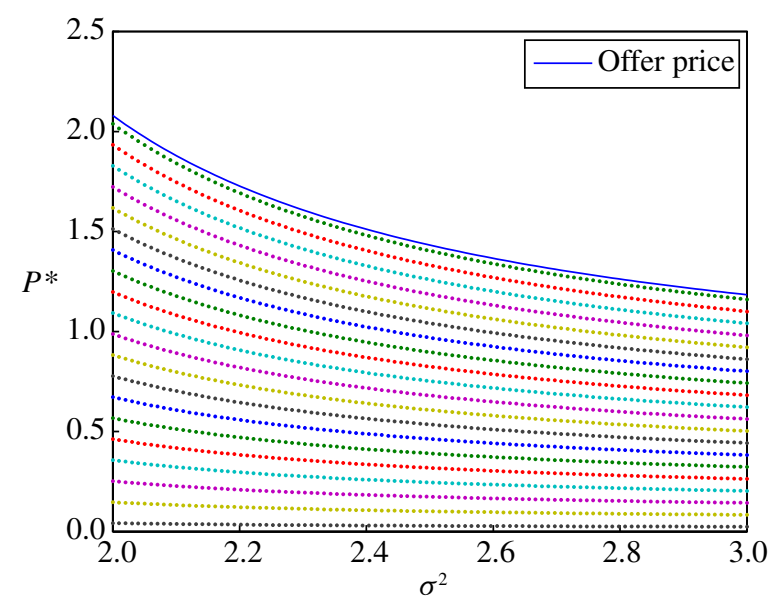

FIGURE 3: Feasible price area for $a=0.5$ and $b=0$. 


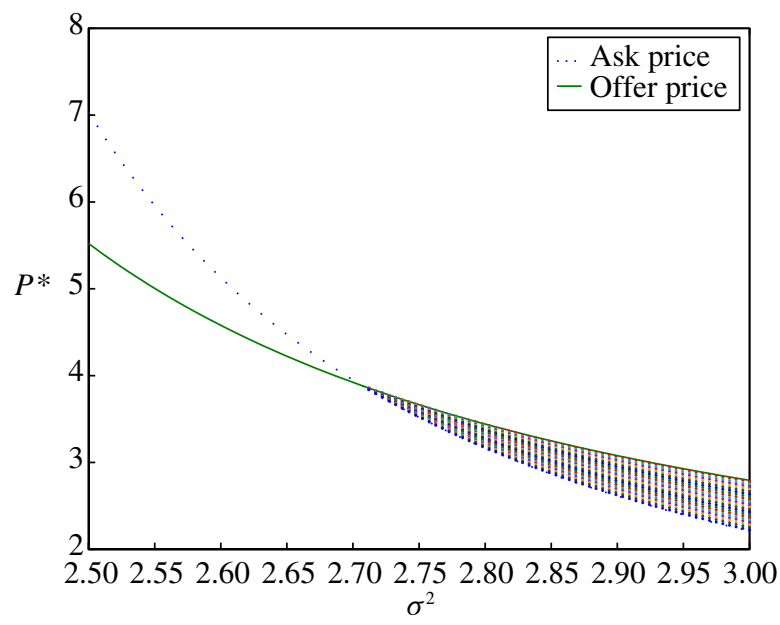

FIGURE 4: Feasible price area for $a=0.5$ and $b=0.5$.

As we can see in Figures 2-4, the volatility parameter $\sigma^{2}$ plays an interesting role. It can either increase or decrease the size of the range of feasible prices.

\section{Conclusion}

We have studied a continuous-time financial market game in which agents possess different levels of information within an anticipative stochastic calculus framework. Technically, our game represents an anticipative stochastic differential game. To the best of the authors' knowledge, such games have not been studied before. We derived necessary and sufficient conditions for the existence of Nash equilibria in this game and studied the impact the level of information has on the Nash equilibria and on general welfare. In the second part we extended the game with two prestages in which information can be traded among the agents. The question of pricing information has so far only been studied in a representative agent framework. We introduced the notion of an equilibrium price for specified information and derived a certain set of inequalities which characterize it. Various examples for the case of initially enlarged filtration were given.

\section{Acknowledgement}

The first author acknowledges support from the Australian Research Council, under grant DP1095969.

\section{References}

Biagini, F. And ØKsendal, B. (2005). A general stochastic calculus approach to insider trading. Appl. Math. Optimization 52, 167-181.

Corcuera, J. M., Imkeller, P., Kohatsu-Higa, A. and Nualart, D. (2004). Additional utility of insiders with imperfect dynamical information. Finance Stoch. 8, 437-450.

EWALD, C.-O. (2005). Optimal logarithmic utility and optimal portfolios for an insider in a stochastic volatility market. Internat. J. Theoret. Appl. Finance 8, 301-319.

HirshleIfER, J. (1971). The private and social value of information and the reward of inventive activity. Amer. Econom. Rev. 61, 561-574. 
IMKELler, P. (2003). Malliavin's calculus in insiders models: additional utility and free lunches. Math. Finance 13, 153-169.

JACOD, J. (1985). Groissement initial, hypothese (H'), et théorème de Girsanov. In Groississements des Filtrations: Exemples et Applications (Lecture Notes Math. 118), eds T. Jeulin and M. Yor, Springer, Berlin, pp. 15-35.

Karatzas, I. And Shreve, S.E. (1988). Brownian Motion and Stochastic Calculus. Springer, New York.

Kohatsu-Higa, A. And Sulem, A. (2006). Utility maximization in an insider influenced market. Math. Finance 16, $153-179$

León, J. A., Navarro R. AND Nualart, D. (2003). An anticipating calculus approach to the utility maximization of an insider. Math. Finance 13, 171-185.

Nualart, D. (1995). The Malliavin Calculus and Related Topics. Springer, New York.

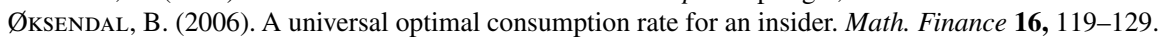

Pikovsky, I. And Karatzas, I. (1996). Anticipative portfolio optimization. Adv. Appl. Prob. 28, 1095-1122.

Russo, F. ANd Vallois, P. (1993). Forward, backward and symmetric stochastic integration. Prob. Theory Relat. Fields 97, 403-421.

Russo, F. ANd Vallois, P. (2000). Stochastic calculus with respect to continuous finite quadratic variation processes. Stoch. Stoch. Reports 70, 1-40. 